\title{
Intraorbital wooden foreign body: A case report
}

\section{Sitaula S', Gautam V 2}

'Sanjeeta Sitaula, Lecturer, BP Koirala Lions Centre for Ophthalmic Studies, Kathmandu, Nepal; 2Vijay Gautam, Ophthalmologist, Sagarmatha Chaudhary Eye Hospital, Lahan, Nepal, Eastern Regional Eye Care Programme.

\begin{abstract}
Intraorbital foreign bodies are one of the commonly encountered orbital problems. They may be sight threatening due to the severity of injury or its complications and even life threatening if not managed appropriately. We present a case of a 24-year old female with history of fall injury from a tree with an impacted intraorbital wooden foreign body. The patient was treated with prophylactic intravenous antibiotics and prompt removal of the foreign body was done under general anaesthesia in the operation theatre. The post operative visual recovery was excellent with significant improvement of ocular motility and limited improvement of ptosis. Hence even organic foreign bodies can have good prognosis with timely intervention.
\end{abstract}

Key words: Computed tomography, Orbital foreign body, Wooden foreign body

\section{INTRODUCTION}

P enetrating orbital injuries are usually results of high velocity injury such as gunshot or industrial injuries ${ }^{1,2}$. However, in a country like Nepal, wooden foreign bodies following low velocity injury is quite common. Organic foreign bodies like wood are notorious for causing cellulitis and abscesses which may further spread to cause central nervous system infections. Hence timely removal of such foreign bodies in a controlled environment like an operation theatre after radiological investigation is beneficial. Here we report a case of an orbital wooden foreign body that was managed in a tertiary level eye hospital in Nepal.

\section{CASE REPORT}

We report a case of 24 year old female from a remote village in Nepal with accidental entry of a piece of wood into the right orbit. The patient fell from a tree from a height of about six metres following which she had pain in the right eye along with inability to open the right eye. There was no history of seizures or vomiting. Two days later, on the $10^{\text {th }}$ of December, 2012 she presented to our hospital with the orbital foreign body. When we examined the patient, there was a wooden piece impacted firmly in the right orbit through an entry

Address for correspondence

Dr. Sanjeeta Sitaula

Lecturer

BPKLCOS, Tribhuvan University Teaching Hospital, Kathmandu, Nepal

E-mail: sanjeeta8272@gmail.com wound just below the right eyebrow with the edge of the foreign body protruding out. The right eye could not be opened making right eye examination impossible. The left eye examination revealed visual acuity of $6 / 6$ with normal ocular findings including the pupils. Neurological evaluation was normal with the Glasgow Coma Scale (GCS) of 15 . There were no other systemic injuries.

Computed tomography (CT) of the orbit was ordered in the emergency which showed a hypodense, linear, tubular structure in right orbit extending medially towards the apex of contra-lateral eye up to the level of the inferior aspect of the left inferior orbital fissure by breaking the bilateral ethmoid sinus walls. The eyeball was intact.

Neurosurgical and otorhinolaryngological consultations were done and there was no surgical intervention required from their side. The patient was then shifted to the operating room on the same day. Examination under anaesthesia showed a firmly impacted wooden foreign body penetrating the orbit on the right side with an inch of the anterior end protruding out. The entry wound was enlarged by giving an incision in the skin just below the brow margin around four centimetres temporally and two centimetres nasally to increase the exposure and access to the foreign body. Then traction sutures were applied on the skin. Blunt dissection was done to separate the foreign body from the surrounding soft tissue. The depth of the blunt dissection was increased 
as much as possible. The foreign body was then grasped with the help of two artery forceps and was removed completely under general anaesthesia. It was a straight eight centimetre long wooden twig with the bark partially shed off. The wound track was explored for any remnants of the wooden piece or bark. Then the wound was closed in layers with a corrugated drain placed in situ. At the end of the procedure, nasal examination was done by the otorhinolaryngologists to rule out cerebrospinal fluid (CSF) rhinorrhoea or other related problems.

On the first postoperative day the visual acuity was $6 / 24$ in the right eye. The wound site was healthy. There was complete ptosis of the right upper lid and extraocular motility was restricted in all gazes. There was chemosis of conjunctiva. Cornea and anterior chamber examination

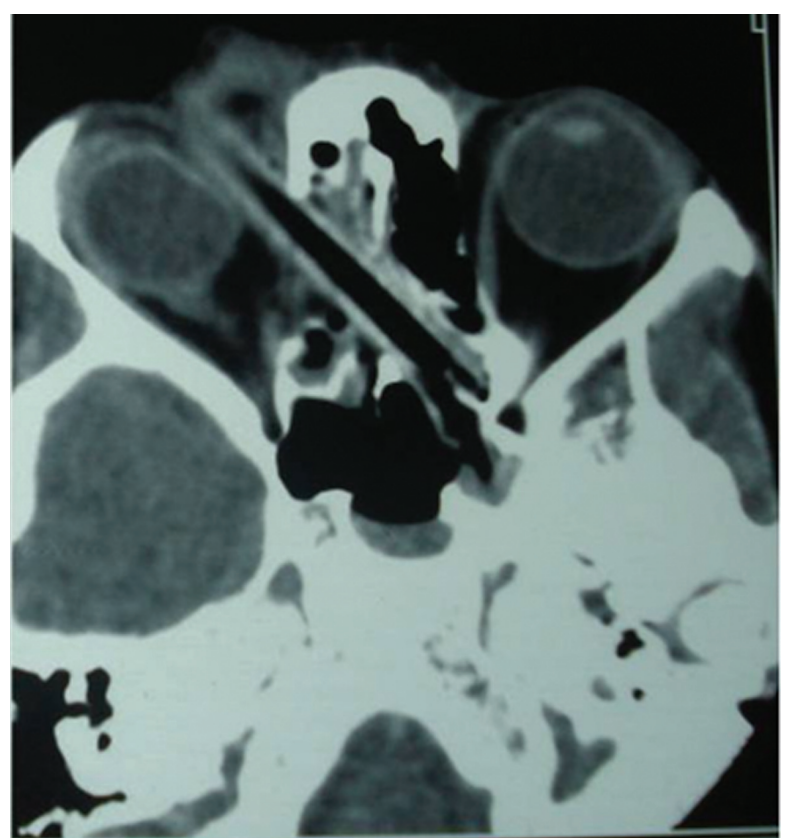

Figure 1: CT-scan of the orbit showing the orbital foreign body

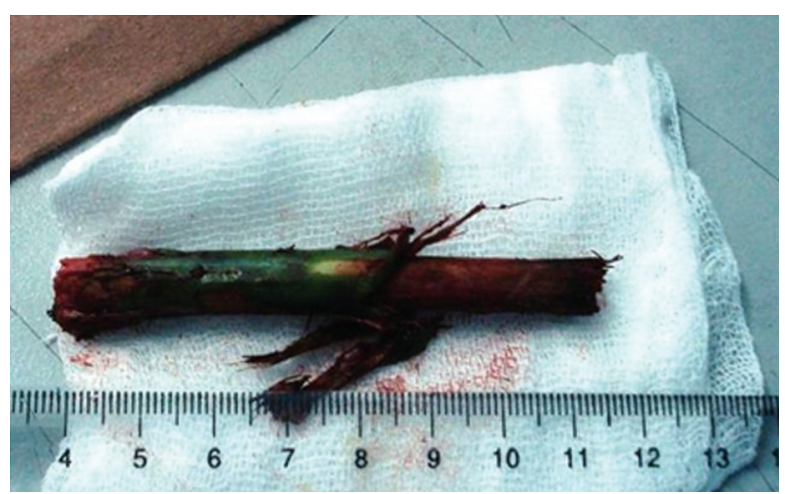

Figure 2: Wooden foreign body removed from the orbit was unremarkable. The pupillary reaction and fundus examination were normal. The patient was managed with intravenous antibiotics and topical antibiotic steroid drops. The patient was monitored in the eye ward for signs of infections and surgical complications. On the third post-operative day the drain was removed as there was minimal serosanguinous discharge. The lid swelling was gradually decreasing and the wound site remained healthy. On the tenth postoperative day the patient was discharged from the eye ward on oral antibiotics. At the time the lid swelling had subsided but there was complete ptosis. The scar was healthy. The extraocular motility was still restricted but the visual acuity had improved to $6 / 18$.

At one month follow up, the visual acuity had improved to $6 / 6$ unaided in both the eyes. The entry wound was visible as a scar that was partially obscured by the eyebrow. The ptosis had improved slightly and the levator action was two $\mathrm{mm}$. There was some amount of extraocular movement visible. Rest of the ocular examination was normal. After that the patient lost to follow up.

\section{DISCUSSION}

The quadrilateral pyramidal shape of the orbit directs foreign bodies either towards the roof of the orbit resulting in frontal lobe injury as when one falls from above or towards the superior orbital fissure as in low velocity injuries ${ }^{3}$. Wooden foreign bodies that have a sharp edge can penetrate deep into the orbit and cranial cavity even through a small entry wound ${ }^{4}$. The resistance of the sclera and the displacement of the globe often protect the eye from perforation ${ }^{5}$. The degree of vision loss depends on the initial injury and the inflammatory response incited by the foreign body and is generally not influenced by the surgical intervention'.

In our case the foreign body had entered from the medial part of the right orbit indenting the right eyeball but leaving it intact, directed posteriorly breaking bilateral ethmoidal sinus walls landing almost upto the superior orbital fissure of the left eye. However fortunately both the optic nerves and the eyeball were spared leading to the the good visual outcome postoperatively.

Various types of orbital foreign bodies have been mentioned in the literature like glass, steel, iron, plastic and wood to mention a few. The inorganic foreign bodies are reported more commonly from the western countries $^{3,6}$, whereas organic foreign bodies and mostly wood are reported from the Indian subcontinent ${ }^{7,8}$. 
Organic foreign body may incite marked inflammatory response due to decay and secondary infections. The risk is particularly high in cases of wooden foreign body as the porous nature of wood acts as a strong growth medium for microorganisms ${ }^{9}$. If the wooden foreign body is missed or some debris remains after removal of foreign body, it may lead to chronic and recurrent orbital inflammation or orbital abscess formation which may spread intracranially forming intracranial abscess or even cavernous sinus thrombosis. They are many such case reports in literature where the initially missed wooden foreign bodies have been discovered later when they presented with complications like recurrent orbital inflammation or sinus with purulent discharge ${ }^{7,10}$. Hence organic foreign bodies require prompt surgical removal in contrast to inert metallic foreign bodies which may be left behind especially in cases of posteriorly located foreign bodies where attempts at surgical removal may be more deleterious to vision ${ }^{6}$.

Imaging studies in the detection of wooden foreign bodies within the orbit have had variable success ${ }^{11}$. Plain x-ray film cannot detect wooden foreign bodies and in computed tomograms the appearance can be misdiagnosed as air especially in missed foreign bodies. Hence MRI scans may be preferred when there is a definite history of wooden foreign body as it is better at detecting them ${ }^{12}$. In our case the foreign body was visible and the $\mathrm{CT}$ scan helped us to visualize the size of foreign body, see the posterior extension and to rule out intracranial injury. All organic foreign bodies need to be removed promptly along with antitetanus prophylaxis and broad spectrum antibiotics covering for anaerobic organisms ${ }^{6}$.

\section{CONCLUSION}

Wooden intraorbital foreign bodies are one of the commonly encountered problem in our setting and prompt surgical removal in the operating room along with preoperative broad spectrum antibiotic prophylaxis can be sight saving as well as life saving.

\section{ACKNOWLEDGEMENT}

We would like to acknowledge Prof Purna Chandra Karmacharya for his surgical assistance and his valuable suggestions.

\section{REFERENCES}

1. Finkelstein $M$, Legmann A, Rubin PA. Projectile metallic foreign bodies in the orbit: a retrospective study of epidemiologic factors, management, and outcomes. Ophthalmology. 1997;104(1):96-103.

2. Michon J, Liu D. Intraorbital foreign bodies. Semin ophthalmol. 1994;9(3):193-9.

3. SchreckingerM, Orringer D, Thompson BG, La Marca F, Sagher O. Transorbital penetrating injury: case series, review of the literature, and proposed management algorithm. J Neurosurg. 2011;114(1):53-61.

4. Mutlukan E, Fleck BW, Cullen JF, Whittle IR. Case of penetrating orbitocranial injury caused by wood. $\mathrm{Br}$ J Ophthalmol. 1991;75(6):374-6.

5. Hansen JE, Gudeman SK, Holgate RC, Saunders RA. Penetrating intracranial wood wounds: clinical limitations of computerized tomography. J Neurosurg. 1988;68(5):752-6.

6. Fulcher TP, McNab AA, Sullivan TJ. Clinical features and management of intraorbital foreign bodies. Ophthalmology. 2002;109(3):494-500.
7. Agarwal PK, Kumar H, Srivastava PK. Unusual orbital foreign bodies. Indian J Ophthalmol. 1993;41(3):1257.

8. Malla G, Bhandari R, Gupta PP, Giri R. Penetrating orbit injury: challenge to emergency medicine. BMC Res Notes. 2013;6:493.

9. Dunn IF, Kim DH, Rubin PA, Blinder R, Gates J, Golby AJ. Orbitocranial wooden foreign body: a pre-, intra-, and postoperative chronicle: case report. Neurosurgery. 2009;65(2):E383-4; discussion E4.

10. Macrae JA. Diagnosis and management of a wooden orbital foreign body: case report. $\mathrm{Br} J$ Ophthalmol. 1979;63(12):848-51.

11. Ho VT, McGuckin JF, Jr., Smergel EM. Intraorbital wooden foreign body: CT and MR appearance. Am J Neuroradiol. 1996;17(1):134-6.

12. Glatt HJ, Custer PL, Barrett L, Sartor K. Magnetic resonance imaging and computed tomography in a model of wooden foreign bodies in the orbit. Ophthal Plast Reconstr Surg. 1990;6(2):108-14. 